\title{
A Review of Adipocytic Tumours, Highlighting the Changing Concepts
}

\author{
Revathy V. J.1 , Krishna Govindan ${ }^{2}$ \\ 1Department of Pathology, Government Medical College, Thiruvananthapuram, Kerala, India. \\ ${ }^{2}$ Department of Pathology, Government Medical College, Thiruvananthapuram, Kerala, India.
}

\section{ABSTRACT}

\section{BACKGROUND}

Adipocytic tumours are the most common soft tissue tumours and are frequently encountered in the routine practice. Vast majority of adipocytic tumours are benign lipomatous tumours, but there are other rare heterogenous adipocytic neoplasms which can create diagnostic difficulties. It can vary from a locally recurrent tumour to a highly malignant type carrying a poor prognosis. The classification of adipocytic tumours has evolved in the last few decades due to advances in the understanding of pathogenetic basis that also provides a greater opportunity for the development of new treatment modalities. So, with the use of ancillary diagnostic tests, definite diagnostic criteria have been established, which have been described in WHO 2013 classification and recently a newer terminology have been described, which is Atypical Spindle Cell Lipomatous Tumour and it is considered as a separate entity based on its morphology and molecular studies. Lipomas are the most common benign adipocytic tumours and the less common tumours are lipoblastoma, angiolipoma, spindle cell/pleomorphic lipoma, myolipoma, chondroid lipoma, lipomatosis of nerve and hibernoma. Well differentiated liposarcomas/ atypical lipomatous tumours are locally aggressive adipocytic neoplasms that do not metastasize. More aggressive liposarcomas include myxoid liposarcoma, pleomorphic liposarcoma and dedifferentiated liposarcomas. Within a time period of 6 months we received 362 adipocytic tumours in the department of Pathology, of which 354 were benign and 8 cases were intermediate (locally aggressive) and malignant which includes atypical spindle cell lipomatous tumour, atypical lipomatous tumour/ well differentiated liposarcoma, myxoid liposarcoma and dedifferentiated liposarcoma. The cases of atypical lipomatous tumour and atypical spindle cell lipomatous tumour, its histological and molecular basis will be discussed along with this review of literature. Atypical spindle cell lipomatous tumours are seen usually in the subcutaneous location with a wide anatomic distribution and more commonly seen in the adults of $6^{\text {th }}$ decade. Gross appearance ranges from yellowish to whitish depending on the amount of adipocytic and fibrous differentiation. Microscopic study shows a hypercellular to a hypocellular lesion with mild to moderately atypical spindle cells, adipocytes, lipoblast, and occasional bizarre, hyperchromatic cells. The stroma can be myxoid or collagenous. Mitotic figures are sparse without any tumour necrosis. Rare heterologous differentiation and different growth patterns have been described including solitary fibrous tumour (SFT) like pattern, angiofibroma like pattern, myxoid liposarcoma like pattern and pericytic pattern. Immunohistochemistry studies show variable expression of CD34, S100 and desmin. Molecular studies identified a characteristic loss of nuclear RB gene expression with deletion of 13q14. The indolent behaviour of atypical spindle cell lipomatous tumour, with very low recurrence rate, no risk of dedifferentiation and no reported distant metastasis / death from disease, highlights the importance of distinguishing it from atypical lipomatous tumour/well differentiated liposarcoma, in order to avoid aggressive surgical resections. So atypical spindle cell lipomatous tumour is now considered as an independent entity rather than a morphological variant of atypical lipomatous tumour.

\section{KEY WORDS}

Adipocytic Tumours, Genomic Alteration
Corresponding Author: Dr. Krishna Govindan, Professor and HOD, Department of Pathology, Government Medical College, Thiruvananthapuram, Kerala, India. E-mail: drkrishnagovindan@gmail.com

\section{DOI: $10.14260 /$ jemds/2020/487}

How to Cite This Article: Revathy VJ, Govindan K. A review of adipocytic tumours, highlighting the changing concepts. J. Evolution Med. Dent. Sci. 2020;9(31):2246-2252, DOI: 10.14260/jemds/2020/487

Submission 24-02-2020,

Peer Review 25-06-2020,

Acceptance 03-07-2020,

Published 03-08-2020.

Copyright (C) 2020 JEMDS. This is an open access article distributed under Creative Commons Attribution License [Attribution 4.0 International (CC BY 4.0)] 


\section{BACKGROUND}

Adipocytic tumours are frequently encountered in routine practice. Vast majority being benign lipomatous tumour, but there are other rare heterogenous adipocytic neoplasms which can create diagnostic difficulties.(1) The classification of adipocytic tumours have evolved in the last decades due to advances in the understanding of pathogenetic basis. Use of molecular cytogenetic techniques like fluorescence in situ hybridization (FISH) improved the accuracy of detecting nonrandom chromosomal translocation and/or other rearrangements in adipocytic tumours. (2) So with the use of ancillary diagnostic tests, definite diagnostic criteria are established, that has been described in WHO 2013 classification and a newer entity have been described recently which is Atypical Spindle Cell Lipomatous Tumour. Our aim is to illustrate the new terminologies and entity with examples.

Adipocytic tumours are diagnosed primarily based on the clinical features and histologic patterns. Immunohistochemistry plays only a little role in the differential diagnosis of adipocytic tumours. Minimally invasive biopsies to diagnose adipocytic tumours have become more common now, so in such cases molecular studies are useful diagnostic adjunct for adipocytic tumours(2),(3),(4)

\section{BENIGN ADIPOCYTIC TUMOURS}

\section{Lipoma}

Which is the most common mesenchymal neoplasm in adults.(5) It can occur at any site, most commonly in the $4^{\text {th }}$ to $6^{\text {th }}$ decade of life. $5 \%$ of cases show multiple lipomas. They arise in the subcutaneous or deep soft tissue including intramuscular or intermuscular sites or can be parosteal.(5) Histologically lipomas are composed of lobules of mature adipocytes, showing variable size and shape. It may show areas of bone formation (osteolipoma), cartilage (chondrolipoma) or fibrous tissue (fibrolipoma).(5) Intramuscular lipoma shows infiltrative growth pattern. Lipoma arborescens shows diffuse fatty infiltration of subsynovial connective tissue.(5) Lipoma stains for S100. $60 \%$ of lipomas shows aberrations in 12q13-15 region, where HMGA2 gene is located.(2),(3)

\section{Lipomatosis}

It is a diffuse overgrowth of mature adipose tissue and involves the trunk, head and neck, abdomen and pelvis. Macroscopy shows poorly circumscribed aggregates of adipose tissue. Microscopy shows lobules/ sheets of adipocytes that infiltrate adjacent structures.(5) Massive accumulation of fat in the neck region may cause death due to laryngeal obstruction. Lipomatosis of nerve is characterized by the expansion of epineurium by adipose and fibrous tissue. The tissue grows between and around the nerve bundles and cause enlargement of the affected nerve.(5)

\section{Chondroid Lipoma}

They are typically deep-seated tumours, involving the skeletal muscle, deep fibrous connective tissue or deep subcutaneous fat. Chondroid lipoma are predominantly seen in middle aged adults. Mostly seen in the proximal extremities and limb girdles.(5) It is a distinctive tumour composed of variable proportion of mature adipose tissue intermingled with lipoblast in a background of chondroid-myxoid matrix.(5) The lipoblast may show range of differentiation from undifferentiated bland cells with minimal cytoplasm to small univacuolated and multivacuolated lipoblasts with scalloped bland nuclei. Chondroid lipoma shows a recurrent translocation in $\mathrm{t}(11 ; 16)(\mathrm{q} 13 ; \mathrm{p} 13)$ which results in the fusion of C11orf95 and MLK genes.(2),(3) Mature adipocytic component show strong positivity for S100.(5)

\section{Spindle Cells / Pleomorphic Lipoma}

These are circumscribed subcutaneous lesions typically occurring in the neck and upper back in older males. Pleomorphic lipoma is said to be the other end of spectrum.(5) The tumour manifest as a long-standing subcutaneous mass in most of the cases. Macroscopy varies from yellowish to grey white depending on the extent of the fatty and spindle cell components.(5) Microscopically, spindle cell lipoma is composed of adipocytes intervened by benign looking spindle cells and collagen bundles in a myxoid matrix, whereas pleomorphic lipoma shows multinucleated floret - like giant cells which is its characteristic feature.(2) Variants include fatdominant tumours with scant spindle cells and others with prominent spindle cell component with few adipocytes.(5) The spindle cells are CD34 positive. The current cytogenetic aberrations appear to be monosomy 13 or deletion of 16q22.(2),(3),(5)

\section{Hibernoma}

It is a rare, benign encapsulated and richly vascularized adipose tissue tumour which is composed of brown fat cells admixed with white adipose tissue(5). These tumours are predominantly seen in the scapular region, thigh, upper extremity and head and neck. It is a painless slow growing tumour.(5) Microscopy shows brown fat cells with granular, multivacuolated cytoplasm and a small central nucleus. Morphological variations include hibernoma with myxoid stroma or a spindle cell component, with thick bundles of collagen fibres, scattered mast cells and brown fat cells. ${ }^{(5)}$ They are characterized by structural rearrangements involving the long arm of chromosome 11(2),(3) Adipocytes are S100 positive and spindle cell component, if present shows CD34 positivity.(5) Hibernoma is a tumour with significant potential for recurrence after local excision.(5)
Lipoblastoma
Lipoblastoma is predominantly seen in children less than 3 years of age. It can be localized as lipoblastoma or diffuse as lipoblastomatosis and they resemble fetal white adipose tissue.(2),(3) They are most commonly seen in the extremities and trunk. Diffuse lipoblastoma originates in the deep soft tissue and is infiltrative.(5) Macroscopy shows a soft, lobulated, yellow, white or tan mass with myxoid nodules or cystic spaces. (5) Microscopically, lipoblastoma shows a lobular appearance which is composed of mature adipocytes separated by thin fibrous septa and lipoblasts in different 
stages of maturation, in a myxoid background and also shows plexiform vascular pattern. It may show a zonal pattern of maturation with immature myxoid cells at the periphery and mature adipocytes in the centre.(5) They are characterized by rearrangements of 8q11-13 involving PLAG1 gene.(2),(3) Immunohistochemistry shows S100 positivity for adipocytes and primitive mesenchymal cells may show reactivity for desmin.(5)

\section{Angiolipoma}

It presents as subcutaneous painful nodules predominantly seen in younger age groups. It can be seen as a single lesion or multiple lesions which are more common. They are most commonly seen in the forearm. Microscopically angiolipomas are composed of lobules of adipocytes separated by thin walled capillary-sized vessels, containing fibrin thrombi (2) (3). The relative proportion of adipocytes and vessels varies and some lesions are entirely composed of vascular channels(5).
\end{abstract}

\section{Extra Renal Angiomyolipoma}

Angiomyolipoma is an uncommon tumour composed of mature adipocytes, spindle cells, smooth muscle cells and thick-walled blood vessels in varying proportions. It is most commonly seen in the kidneys. It is also reported in extrarenal sites. It also has an association with tuberous sclerosis. Immunohistochemistry shows both melanocytic and smooth muscle marker positivity, which include HMB45 (melanocytic) and smooth muscle actin and muscle- specific actin(2) (3).

\section{Extra Adrenal Myelolipoma}

It is a rare tumour. Myelolipoma is most common in the adrenal gland, but extra adrenal sites are also described. Microscopically, it shows mature fat cells and hematopoietic elements composed of erythroid series, myeloid series and megakaryocytes.(2), (3)

\section{Myolipoma}

It is an extra-uterine tumour composed of mature adipose tissue and smooth muscle. It is an extremely rare tumour, occurring in adults and is most commonly seen in females. It present as a deep seated mass in abdominal cavity, retroperitoneum or inguinal region.(5) Microscopy shows a well circumscribed or encapsulated lesion with thin fascicles of bland smooth muscle traversing mature adipocytes.(5) Desmin and SMA positivity confirms smooth muscle differentiation. Molecular studies showed HMGA2 gene alterations.(5)

\section{Evolving Concepts}

The last edition of WHO manual of soft tissue and bone tumours in 2013 classify the adipocytic tumours into benign, intermediate (locally aggressive) and malignant. Of which, the intermediate (locally aggressive) type includes atypical lipomatous tumour/ well differentiated liposarcoma.(5)

The well differentiated liposarcoma includes $50 \%$ of all liposarcomas.(6) Definite criteria have been established based on the tumour location and surgical resectability to classify liposarcomas histopathologically as well differentiated liposarcoma (WDLS) or as an atypical lipomatous tumour (ALT), which represent a locally aggressive tumour with no risk of metastasis. ${ }^{(7),(8)}$ This reflects the course of the disease regarding the incidence of recurrence, dedifferentiation and distant metastasis.(6) If the tumour is seen in the extremities, where a complete surgical excision is achievable, it is termed as atypical lipomatous tumour, where surgery is the curative treatment. If the tumours located in retroperitoneum, mediastinum or sites where a wide local excision is not possible, is termed as well-differentiated liposarcoma, which shows a high incidence of local recurrence(6),(7),(9),(10) The defining genetic feature of both well differentiated liposarcomas and atypical lipomatous tumour is the presence of ring or giant marker chromosome that contain amplification of the 12q13-15 region, that represents amplified oncogenes MDM2, CDK-4, CPM genes.(5),(7),(11)

Both atypical lipomatous tumour and well differentiated liposarcomas are composed entirely/ partly of mature adipocytes with significant variation in cell size. Focal nuclear atypia can be seen in both adipocytes and stromal cells.(5) They may show mono vacuolated or multivacuolated lipoblasts.(12) Different subtypes have been mentioned which includes adipocytic, sclerotic and inflammatory subtypes, with all the three showing similar molecular genetic study of MDM2/CDK4 amplification.(9) Another variant which is a spindle cell subtype was mentioned under atypical lipomatous tumour/ well differentiated liposarcoma, which shows spindle cells in addition to the adipocytic component with nuclear atypia in both adipocytes and spindle cells. But the molecular study of this subtype revealed a loss of RB gene expression and consistent absence of MDM2/CDK4 amplification.(9),(12) This led to the introduction of a newer entity, Atypical spindle cell lipomatous tumour.

\section{Atypical Spindle Cell Lipomatous Tumour}

Atypical spindle cell lipomatous tumour presents as an enlarging soft tissue mass, more commonly in the subcutaneous location with a wide anatomic distribution including hand, feet, thigh, shoulder, buttock and rarely retroperitoneum and mediastinum.(1) More commonly seen in the adults of $6^{\text {th }}$ decade, with very low tendency for local recurrence if completely excised and there is no risk of dedifferentiation (12).

This lesion is characterized by ill-defined tumour margins, with varying gross appearance ranging from yellowish to whitish cut surface depending on the amount of adipocytic and fibrous differentiation.(12) Microscopic study shows a hypercellular or a hypocellular lesion [Figure 3] with mild to moderately atypical spindle cells, adipocytes, lipoblast, and occasional bizarre, hyperchromatic cells in the spindle or adipocytic component [Figures 1, 2]. The stroma can be myxoid or collagenous.(1),(12) Mitotic figures are sparse with no tumour necrosis. Rarely heterologous differentiation has been reported with smooth muscle, osseous or cartilaginous elements. Different growth patterns are described.(1)

- SFT like pattern- showing stag horn-like vessels in a collagen rich background.

- Angiofibroma like pattern- with prominent branching capillary network. 
- Myxoid liposarcoma like pattern- with abundant myxoid matrix and with prominent chicken wire like capillaries.

- Pericytic type- showing perivascular location of atypical spindle cells.

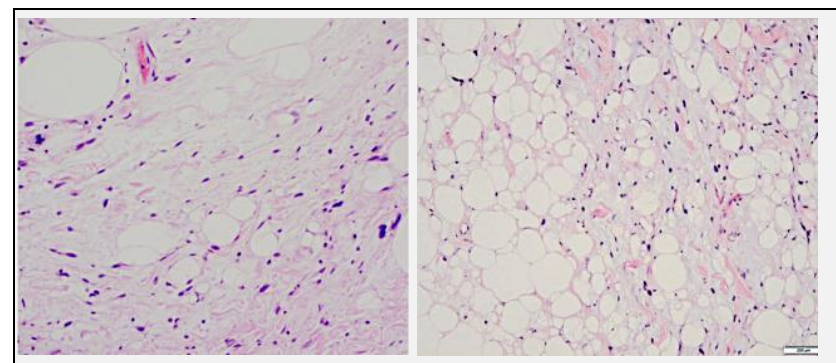

Figure 1a. Adipocytic Component with Scattered Atypical Spindle Cells $^{(1)}$. Figure 1b. Adipocytic Component with Variation in Cell Size ${ }^{(1)}$

(Microscopic Picture taken from "what's new in Adipocytic Neoplasia", by Creytens D.) (Microscopic picture taken from "what's new in Adipocytic Neoplasia", by Creytens D.)
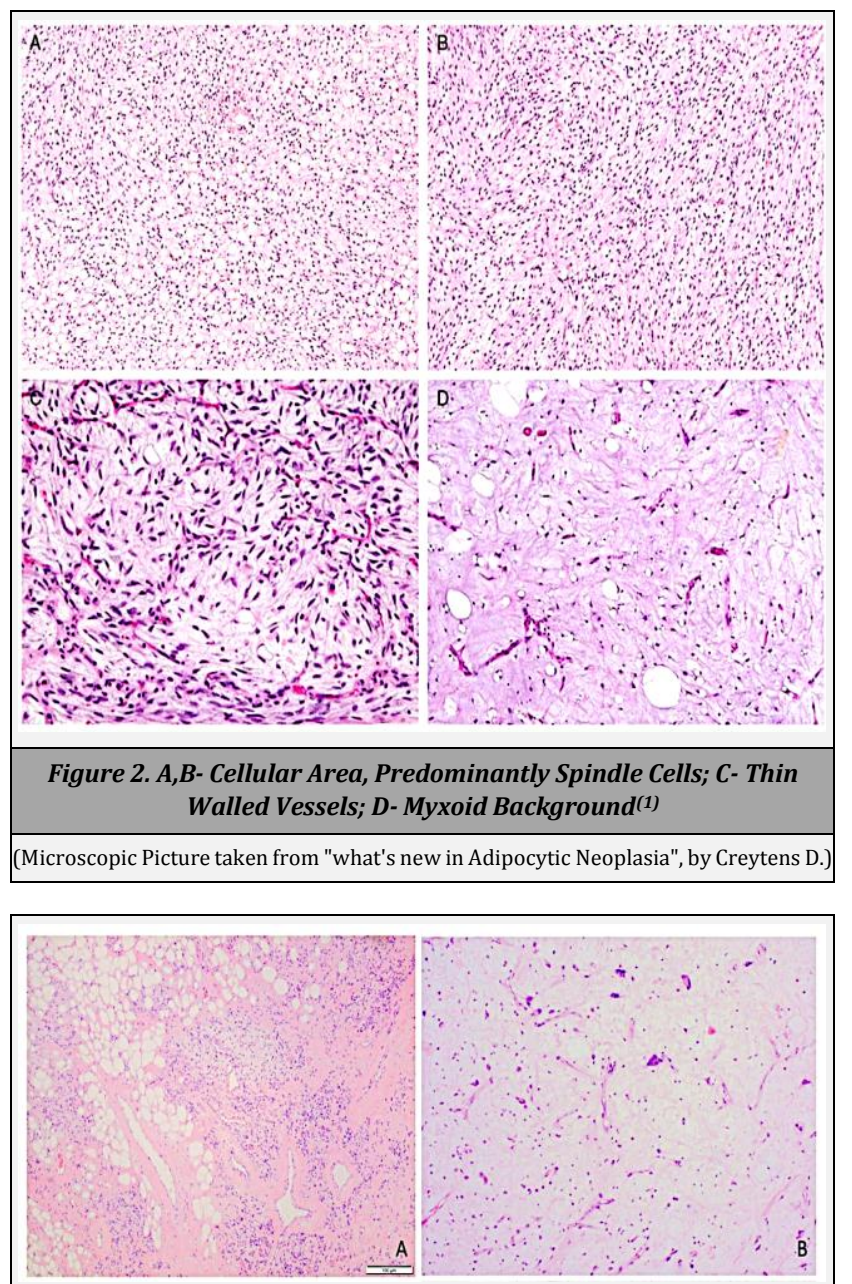

Figure 3. A- Hypercellular Lesion; B-Hypocellular Lesion(1)

(microscopic picture taken from "what's new in adipocytic neoplasia", by Creytens D.)

Immunohistochemistry study shows variable expression of CD34, S100 and desmin. Focal nuclear positivity for MDM2 or CDK4 expression, but never both together.(12) Molecular study shows characteristic loss of nuclear RB gene expression in $50-70 \%$ cases with deletion or loss of $13 q 14$ and consistent absence of MDM2/CDK4 amplification.(1),(12) This entity shows an excellent prognosis, if completely excised with a very low recurrence rate of nearly $10 \% .^{(12)}$

\section{MALIGNANT ADIPOCYTIC TUMOURS}

\section{Dedifferentiated Liposarcoma}

This is a malignant adipocytic neoplasm which shows transition from atypical lipomatous tumour/well differentiated liposarcoma into a non-lipogenic sarcoma in an abrupt fashion and shows varying histologic grade(5). $90 \%$ of the dedifferentiated liposarcomas occur de novo while only $10 \%$ occur in recurrent cases.(2),(3) Predominantly seen in elderly and common sites include retroperitoneum and extremities. (2) The risk of dedifferentiation is more common in deep seated lesions and around $15-20 \%$ cases show metastasis. $90 \%$ of dedifferentiated liposarcomas shows high grade poorly differentiated areas and may mimic sarcomas of different origin. Low grade dedifferentiation, which are rare, is characterized by the presence of uniform fibroblastic spindle cells with mild nuclear atypia.(5) Dedifferentiated liposarcomas may show heterologous differentiation with myogenic or osteosarcomatous or chondrosarcomatous areas. Similar to atypical lipomatous tumour or well differentiated liposarcoma, dedifferentiated liposarcoma shows the presence of giant or ring marker chromosomes.(2),(3) But the peculiarity of dedifferentiated liposarcoma is the presence of multiple abnormal clones. Fluorescence in situ hybridization and comparative genomic hybridization showed the ring and giant marker chromosomes are composed of 12q13-15 amplification involving MDM2, CDK4 and HMGA2.(2),(3) They are characterized by local recurrence in at least $40 \%$ of cases.

\section{Myxoid Liposarcoma / Round Cell Liposarcoma}

Previously it was considered as two distinct subtypes. Myxoid liposarcomas are the second most common subtypes of liposarcomas. It represents about $5 \%$ of all soft tissue sarcomas in adults. It is predominantly seen in young adults and equally common in males and females. The most common site is the deep soft tissue of extremities. Microscopically, it has a nodular growth pattern with enhanced cellularity at the periphery of the lobules.(5) It is composed of uniform round to oval shaped primitive (non - lipogenic) mesenchymal cells and varying number of signet ring cell lipoblasts in a myxoid background, with a characteristic delicate, arborizing, chicken-wire capillary vasculature. In contrast to other types of liposarcomas, myxoid liposarcoma metastasize to unusual sites like retroperitoneum, opposite extremity or axilla. A subset of myxoid liposarcoma shows histological progression to round cell morphology, which has a poor prognosis.(5) The high grade (round cell) areas are characterized by back to back primitive round cells with a high nuclear to cytoplasmic ratio, with no intervening myxoid stroma. ${ }^{(5)}$ More than $90 \%$ of cases shows a translocation $\mathrm{t}(12 ; 16)(\mathrm{q} 13 ; \mathrm{p} 11)$, resulting in a FUSDDIT3 fusion gene and the rest shows $t(12 ; 22)(q 13 ; q 12)$ resulting in EWSR1- DDIT3 fusion gene.(2),(3),(5)

\section{Pleomorphic Liposarcoma}

It is a rare malignant neoplasm with adipocytic differentiation showing pleomorphic lipoblasts. It is predominantly seen in elderly patients and is usually deep seated. The most common site is extremities.(5) This is a high grade aggressive neoplasm with $30-50 \%$ of cases showing metastasis. Microscopically it is an infiltrative neoplasm composed of pleomorphic spindle 
cells, pleomorphic multivacuolated lipoblasts and multinucleated giant cells.(2),(3) The presence of lipoblast is necessary for the diagnosis. It shows variable mitotic rate, with a median of 19-25 mitoses per 10 HPF. Necrosis is present in more than half of the cases.(5) Lipoblast shows variable staining for S100. Pleomorphic liposarcomas are associated with highly complex karyotypes which lack specific structural or numerical aberrations.(2),(3) MDM2-CDK4 amplification is typically absent, that helps to distinguish pleomorphic liposarcoma from dedifferentiated liposarcoma.(5)

A six months statistics regarding the cases of adipocytic tumours received in the department of pathology, government medical college, Thiruvananthapuram showed 362 cases. Out of which 354 were benign and 8 cases were intermediate (locally aggressive) and malignant which includes atypical spindle cell lipomatous tumour, atypical lipomatous tumour/well differentiated liposarcoma, myxoid liposarcoma and dedifferentiated liposarcoma; which shows that $98 \%$ of cases received were benign tumours [Figure 4].

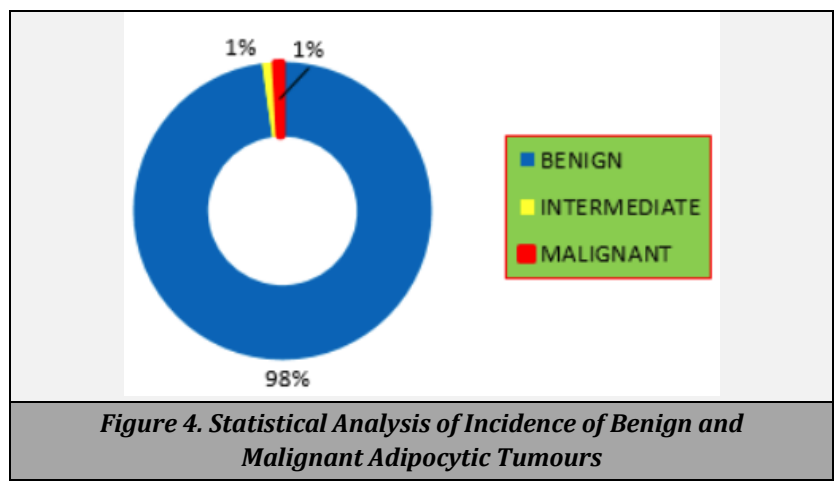

\section{Illustrative Example 1}

A 54-year-old female presented with a right thigh swelling measuring $8 \mathrm{~cm} \mathrm{X} 6 \mathrm{~cm} \mathrm{X} 3 \mathrm{~cm}$ in the medial aspect. MRI showed an oval well defined, T1 T2 hyperintense lesion in the subcutaneous plane with thin septations consistent with lipoma. Gross wide local excision of the tumour showed a fairly circumscribed lesion below the skin, with yellowish lobulated cut surface.

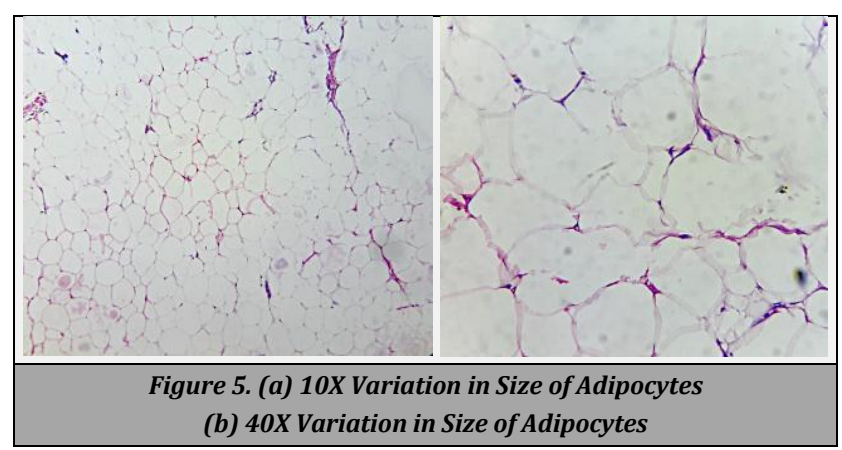

Microscopy showed a neoplasm composed of lobules of adipocytes with varying cell size, separated by thin fibrous septae, with few adipocytes showing nuclear atypia with eccentrically placed, intended vesicular nucleus [Figure 5, 6] and diagnosed as atypical lipomatous tumour as the lesion occurred in extremity with wide local excision giving a good margin clearance. Further fluorescence in-situ hybridization was done and showed a positive MDM2 gene amplification. Patient is under follow-up.

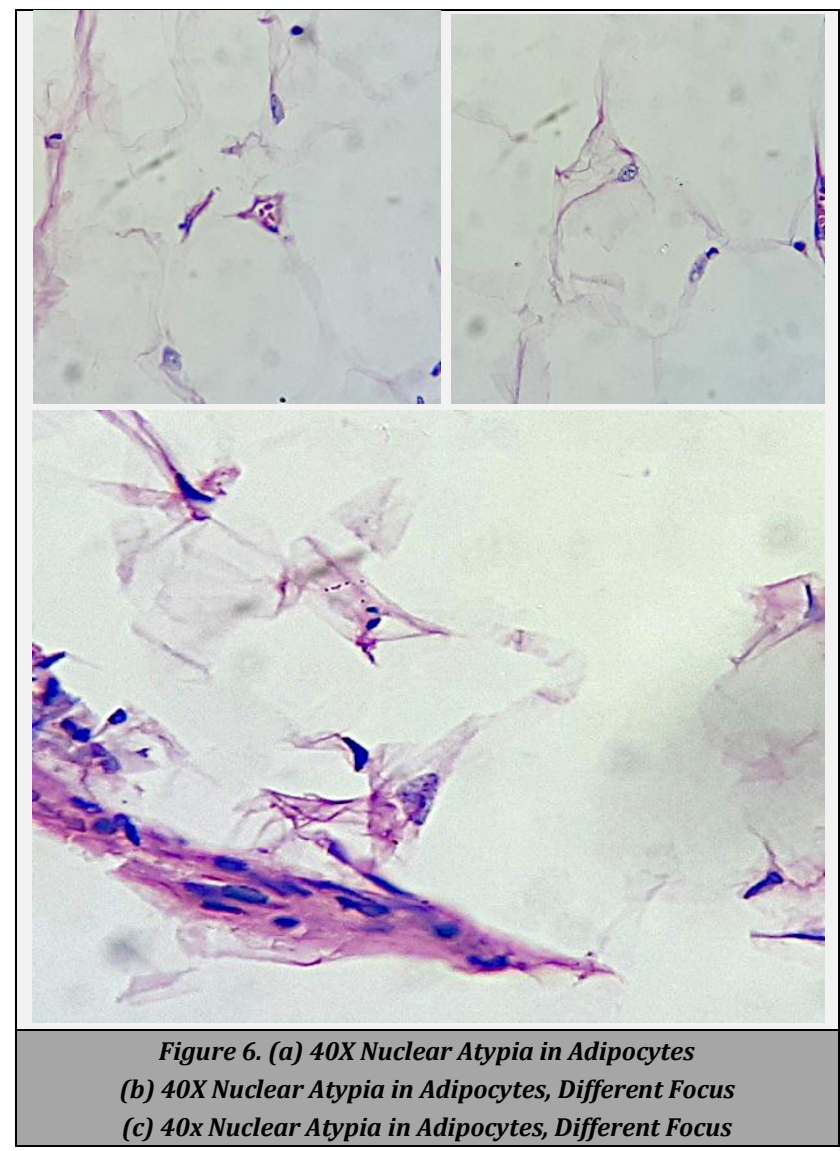

\section{Illustrative Example 2}

A 59-year-old male presented with constipation for past 4 months. On examination per abdomen showed a palpable mass in the umbilical region extending up to the left lumbar region. CT abdomen showed a $14 \mathrm{~cm}$ X $10 \mathrm{~cm} \mathrm{X} 9 \mathrm{~cm}$ hypodense mesenteric space occupying lesion in the left lumbar region with well-defined margins, with a clinical diagnosis of mesenteric liposarcoma.

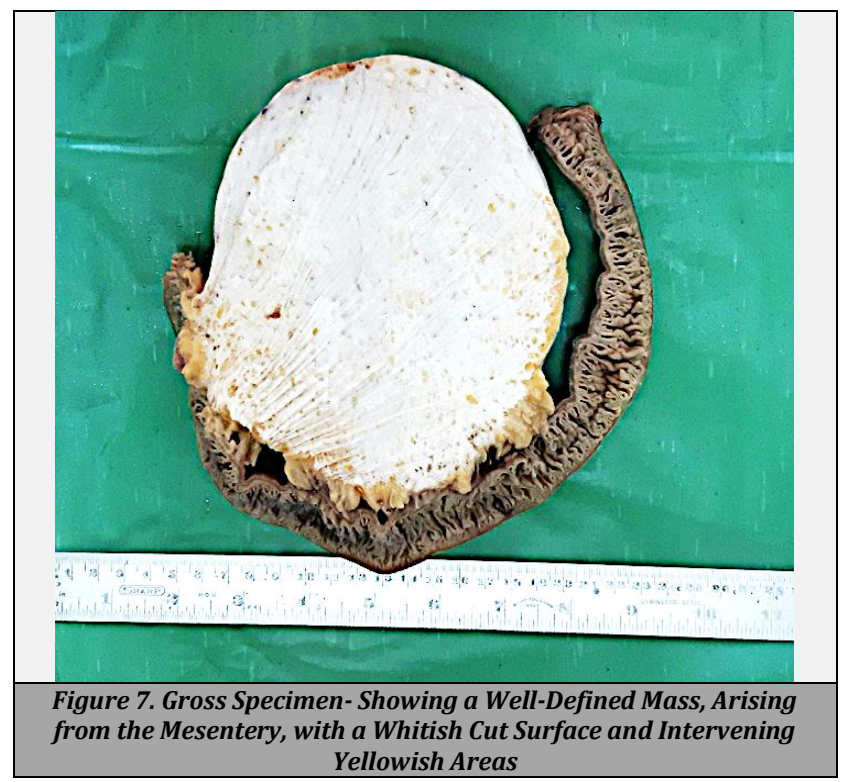



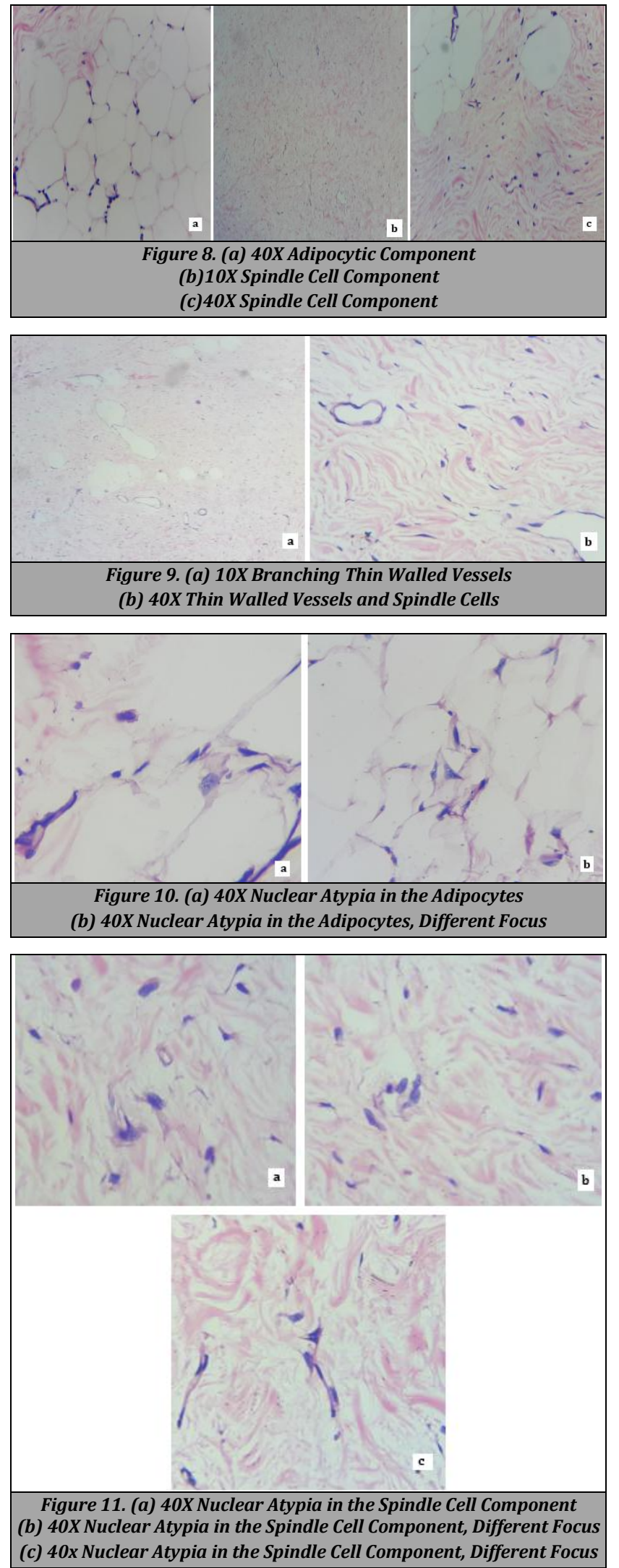

Macroscopy showed a nodular mass in the mesenteric border of small intestine. Lesion appeared to arise from the mesenteric fat, with a whitish cut surface and tiny yellowish adipocytic areas in between [Figure 7]. Microscopy showed a neoplasm composed cells in diffuse sheets. Cells were predominantly spindly with indistinct cell border, elongated vesicular nucleus with fine chromatin. Spindle cells are intervened by collagen fibres. Scattered nests of adipocytes and branching vessels were seen [Figure 8, 9]. Both adipocytic and spindle cell nucleus showed atypia [Figure 10,11]. No mitosis, necrosis or lipoblast were identified.

Immunohistochemistry study of neoplastic spindle cells showed strong cytoplasmic positivity for vimentin and focal positivity for CD34. Spindle cells were negative for SMA, desmin, S100 and STAT-6, which ruled out other fibroblastic and smooth muscle tumours [Figure 12]. Hence diagnosed as Atypical spindle cell lipomatous tumour. Fluorescence in situ hybridization study showed absence of MDM2/CDK4 amplification. Patient is being followed up and there is no recurrence.

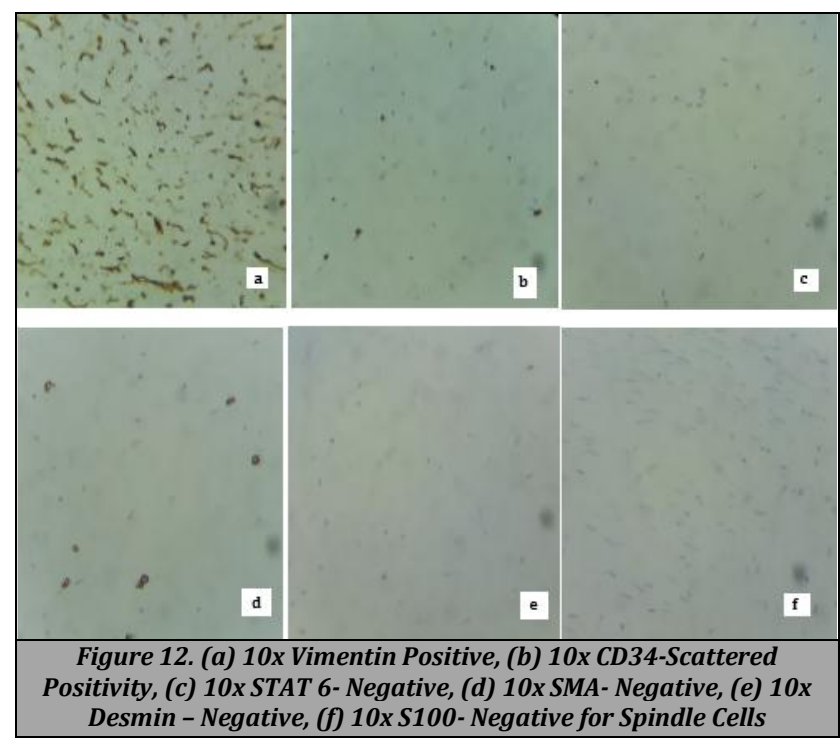

\section{CONCLUSIONS}

The indolent behaviour of atypical spindle cell lipomatous tumour, with very low recurrence rate and no reported distant metastasis / death from the disease, highlights the importance of distinguishing it from atypical lipomatous tumour / well differentiated liposarcoma, in order to avoid aggressive surgical resections. So, atypical spindle cell lipomatous tumour is now considered as an independent entity rather than a morphological variant of atypical lipomatous tumour / well differentiated liposarcoma.

Financial or Other Competing Interests: None.

\section{REFERENCES}

[1] Creytens D. What's new in adipocytic neoplasia? Virchows Arch 2020;476(1):29-39.

[2] Nishio J. Contributions of cytogenetics and molecular cytogenetics to the diagnosis of adipocytic tumors. J Biomed Biotechnol 2011;2011:524067.

[3] Gupta P, Potti TA, Wuertzer SD, et al. Spectrum of fatcontaining soft-tissue masses at MR imaging: the common, the uncommon, the characteristic, and the sometimes confusing. Radiographics 2016;36(3):756-66.

[4] Hameed M. Pathology and genetics of adipocytic tumors. Cytogenet Genome Res 2007;118(2-4):138-47. 
[5] Fletcher CDM, Bridge JA, Hogendoorn PCW, et al. WHO classification of tumours of soft tissue and bone: WHO classification of tumour. $4^{\text {th }}$ edn. Vol. 5. World Health Organization 2013.

[6] Rauh J, Klein A, Baur-Melnyk A, et al. The role of surgical margins in atypical lipomatous tumours of the extremities. BMC Musculoskelet Disord 2018;19(1):152.

[7] Eisenberg B, Nassif NA, Tseng W, et al. Recent advances in the management of liposarcoma. F1000Res 2016;5:2907.

[8] Renne SL. Who, how and what of pathology of soft tissue sarcoma. Chin Clin Oncol 2018; cco.2018.10.09.

[9] Rosai J. Rosai and Ackerman's surgical pathology. 10 $10^{\text {th }}$ edn. Vol. 1. St Louis, Missouri: Mosby/Elsiever 2011: p. 294, 295, 324, 351, 359, 363, 342, 369.
[10] Mentzel T, Palmedo G, Kuhnen C. Well-differentiated spindle cell liposarcoma ('atypical spindle cell lipomatous tumor) does not belong to the spectrum of atypical lipomatous tumor but has a close relationship to spindle cell lipoma: clinicopathologic, immunohistochemical, and molecular analysis of six cases. Mod Pathol 2010;23(5):729-36.

[11] Wei S, Henderson-Jackson E, Qian X, et al. Soft tissue tumor immunohistochemistry update: illustrative examples of diagnostic pearls to avoid pitfalls. Arch Pathol Lab Med 2017;141(8):1072-91.

[12] Schaefer IM, Fletcher CD. Recent advances in the diagnosis of soft tissue tumours. Pathology 2018;50(1):37-48. 